John T. Boyle

\title{
Gastroesophageal reflux disease in 2006
}

\section{The imperfect diagnosis}

Published online: 22 July 2006

C) Springer-Verlag 2006

\begin{abstract}
There continues to be significant controversy related to diagnostic testing for gastroesophageal reflux disease (GERD). Clearly, barium contrast fluoroscopy is superior to any other test in defining the anatomy of the upper gastrointestinal (UGI) tract. Although fluoroscopy can demonstrate gastroesophageal reflux (GER), this observation does not equate to GERD. Fluoroscopy time should not be prolonged to attempt to demonstrate GER during barium contrast radiography. There are no data to justify prolonging fluoroscopy time to perform provocative maneuvers to demonstrate reflux during barium contrast UGI series. Symptoms of GERD may be associated with physiologic esophageal acid exposure measured by intraesophageal $\mathrm{pH}$ monitoring, and a significant percentage of patients with abnormal esophageal acid exposure have no or minimal clinical symptoms of reflux. Abnormal acid exposure defined by $\mathrm{pH}$ monitoring over a $24-\mathrm{h}$ period does not equate to GERD. In clinical practice presumptive diagnosis of GERD is reasonably assumed by substantial reduction or elimination of suspected reflux symptoms during therapeutic trial of acid reduction therapy.
\end{abstract}

Keywords Gastroesophageal reflux disease · Gastroesophageal reflux

In review articles gastroesophageal reflux (GER) appears to be a straightforward problem. In practice, however, there continues to be significant controversy, particularly related to diagnosis. GER is defined as the passage of gastric

\footnotetext{
J. T. Boyle ( $\square)$

Division of Pediatric Gastroenterology,

Children's Hospital of Alabama,

ACC 618, 1600 7th Ave. South,

Birmingham, AL 35233, USA

e-mail: tboyle@uab.edu

\section{J. T. Boyle}

Division of Pediatric Gastroenterology,

University of Alabama-Birmingham School of Medicine,

Birmingham, AL 35233, USA
}

contents into the esophagus. In infants and children reflux may be thought of in one of three patterns. First, intraesophageal $\mathrm{pH}$ monitoring in asymptomatic infants and children has revealed that GER is a silent physiologic phenomenon. If acid reflux is defined as a drop in intraesophageal $\mathrm{pH}$ to less than 4 (a pH at which acid is capable of producing tissue injury), the infant esophagus is exposed to an acid environment for $11 \%$ and the child and adolescent esophagus for 5-6\% of a $24-\mathrm{h}$ period [1]. Thus, all infants and children reflux to some degree. Second, reflux is a common clinical syndrome in infancy manifested by recurrent vomiting or oral regurgitation. In the first 6 months of life, $50-60 \%$ of infants vomit at least once daily, and $15-20 \%$ more than four times daily [2]. That reflux in infancy is a developmental phenomenon is supported by the rapid improvement in frequency of symptoms between the ages of 6 and 12 months. By 1 year of age, $5 \%$ of infants may still vomit once daily, but less than $1 \%$ will vomit in excess of four times a day. Third, when refluxed gastric contents produce clinical symptoms or tissue damage, GER is called a disease, gastroesophageal reflux disease (GERD). In most patients with GERD there is an increased frequency of reflux or prolonged exposure of the esophagus to an acid environment beyond physiologic parameters. However, GERD may also occur in patients with asymptomatic physiologic reflux [3].

The predominant mechanism of reflux in all three patterns is the same: transient relaxation of the lower esophageal sphincter (LES) [4]. The LES is believed to be the major barrier to reflux in humans. It is termed a physiologic sphincter because there is no anatomical structure, such as the pylorus, at the gastroesophageal junction. The smooth muscle in this area behaves as a sphincter in that it maintains a basal tone greater than the esophagus above or stomach below, and relaxes with stimuli from above (swallow or esophageal distension). Reflux is not caused by a weak sphincter, but rather a sphincter that relaxes at times it should not. These transient relaxations are not associated with any esophageal body motor events. Triggers of transient relaxations described to date include: (1) a vagovagal reflex initiated by gastric 
distension, (2) a subthreshold swallow that does not trigger peristalsis, and (3) a vagovagal reflex initiated by cardiopulmonary receptors.

The symptoms of GERD may be classified as esophageal or extraesophageal. In an infant with recurrent vomiting or oral regurgitation the symptoms of esophageal GERD include unexplained irritability, feeding difficulty, poor weight gain, or sleep disturbance. In the older child the symptoms may include chronic heartburn, epigastric abdominal pain, oral regurgitation, episodic vomiting, dysphagia, and rarely hematemesis. Heartburn and/or oral regurgitation are reported to occur in $2 \%$ of children aged 3-9 years, in 5-8\% of children aged $10-17$ years, and in $20 \%$ of adults $[5,6]$. The pathogenesis of esophageal GERD is related to the exposure of the esophagus to gastric contents (increased frequency of reflux and/or impaired esophageal acid clearance), volume, potency, and height of refluxed material, defective tissue resistance, reduced esophageal capacitance, or heightened esophageal sensitivity. The symptom of heartburn does not necessarily mean esophagitis. A patient with heightened esophageal sensitivity may have as much or more heartburn as a patient with erosive esophagitis. Extraesophageal symptoms of GERD include chronic cough, wheezing/asthma, apnea, bradycardia, chronic sore throat, hoarseness, dental erosions, and recurrent otitis/sinusitis [7]. The pathogenesis of extraesophageal GERD may include: (1) regurgitation into the oral pharynx or nasal passages causing direct caustic injury, inflammation, edema, eustachian tube dysfunction, or impaired sinus drainage; (2) microaspiration resulting in direct caustic injury, inflammation, edema, epithelial hypertrophy, laryngitis, pneumonitis, or vagal reflex-mediated cough, laryngospasm, or bronchospasm; or (3) stimulation of esophageal vagal afferent receptors resulting in reflux-mediated change in airway resistance or bronchial hyperreactivity.

Options for the treatment of GERD include lifestyle changes, pharmacotherapy, and surgical therapy. Lifestyle changes include avoiding overfeeding, thickened feeds, upright positioning during sleep, and avoidance of secondhand smoke. The obvious goal of pharmacotherapy would be to reduce transient LES relaxations. However, to date, there are no approved medications that target the actual mechanism of reflux. Pharmacologic agents that have been reported to decrease the frequency of transient relaxations include atropine, cholecystokinin antagonist receptors, nitric oxide synthase inhibitors, and GABA receptor antagonists (e.g. baclofen). The goal of current pharmacologic therapy of GERD is to reduce esophageal acid exposure either by reducing gastric acid secretion or enhancing GI motility to accelerate gastric emptying or hasten esophageal acid clearance following reflux [3]. Both histamine receptor antagonists $\left(\mathrm{H}_{2} \mathrm{RAs}\right.$, e.g. ranitidine, famotidine, cimetidine, and nizatidine) and proton pump inhibitors (PPIs, e.g. omeprazole, lansoprazole) have been reported to be efficacious in the treatment of pediatric GERD. Only in erosive esophagitis have PPIs been found to be superior to $\mathrm{H}_{2} \mathrm{RAs}$. Whereas the traditional approach to acid-reducing therapy in adults is step down (begin treatment with PPI $\rightarrow$ maintain improvement with PPI $\rightarrow$ step down to an $\mathrm{H}_{2} \mathrm{RA}$ ), the approach in children has traditionally been step up (begin treatment with $\mathrm{H}_{2} \mathrm{RA} \rightarrow$ if inadequate response step up to PPI $\rightarrow$ if inadequate response increase PPI dose). As a general rule, there is no evidence-based justification for the routine use of currently available promotility agents (metoclopramide, bethanecol, erythromycin) to treat GERD in children. The principles of antireflux surgery include lengthening the intraabdominal segment of the esophagus, approximating the diaphragmatic crura, and wrapping the gastric fundus around the LES to reinforce the antireflux barrier. Unlike pharmacotherapy, antireflux surgery has been reported to decrease the frequency of transient LES relaxations.

Several principles must be considered in choosing GERD therapy. It is very important to identify outcome variables before initiating therapy (i.e. heartburn, unexplained irritability in an infant, asthma outcome parameters such as quality of life indexes, pulmonary function tests, hospitalizations, need for steroids). Unlike esophageal symptoms of GERD, extraesophageal symptoms require higher doses of acid-reduction therapy for prolonged periods (3-6 months) before assessing outcome variables [8]. Finally, treatment requirements for the same esophageal symptoms differ between individual patients. Many patients require only situational medication (antacid overthe-counter acid-reducing agents when eating spicy foods), while others require more prolonged intermittent therapy (2-8 weeks of acid-reducing agents), and still others require continuous acid reducing agents.

\section{Diagnosis of GERD}

In clinical practice a presumptive diagnosis is reasonably assumed by a substantial reduction or elimination of suspected reflux symptoms during a therapeutic trial of acid-reduction therapy. However, many clinicians prefer an objective test to confirm a diagnosis of GERD in an infant or child with chronic vomiting, symptoms that suggest esophageal pain, or respiratory disease that is not responding to the usual therapies. The presumptive diagnostic tests for GERD include barium contrast upper GI series, intraesophageal $\mathrm{pH}$ monitoring, multichannel intraluminal impedance, scintigraphy of a technetium-sulfur colloid test meal, and upper endoscopy with esophageal biopsy to detect esophagitis. Intraesophageal $\mathrm{pH}$ monitoring has long been considered the gold standard since the test allows direct measurement of esophageal $\mathrm{pH}$ in physiologic circumstances over many hours $[9,10]$. Prolonged $\mathrm{pH}$ monitoring determines the frequency of reflux and the percent time that the esophagus is exposed to an acid environment over a defined time period, detects silent reflux in the absence of vomiting or oral regurgitation, assesses the height of reflux in the esophagus, determines effectiveness of esophageal clearance mechanisms, determines if a temporal association between acid reflux and symptoms exist, and assesses the adequacy of $\mathrm{H}_{2} \mathrm{RA}$ or PPI dosage in unresponsive patients. 
With new wireless technology a $\mathrm{pH}$ probe can be secured endoscopically into the esophageal wall to allow continuous ambulatory monitoring over $48 \mathrm{~h}$ [11]. Prolonged intraesophageal $\mathrm{pH}$ monitoring has a high specificity (about 95\%) meaning that patients without GERD are likely to have a negative test. In both adult and pediatric patients with erosive esophagitis, $\mathrm{pH}$ monitoring has a high sensitivity (90-95\%). However, in adults with symptoms of GERD but without gross endoscopic esophagitis, the sensitivity of $\mathrm{pH}$ monitoring drops to about $50 \%$ [12]. Approximately $40 \%$ of adults with symptoms of chronic heartburn, acid regurgitation, chest pain, belching, odynophagia, or pharyngeal pain will have a negative $\mathrm{pH}$ probe study. Infants and children with GERD rarely have visual changes of esophagitis at upper endoscopy. That $\mathrm{pH}$ monitoring has a low sensitivity for diagnosing GERD in the majority of pediatric patients is suggested by two Scandinavian studies in which only $29-49 \%$ of asthmatic children with clinical symptoms of GERD had positive $\mathrm{pH}$ studies $[13,14]$. These observations contrast with a number of case series in which $50 \%$ of asthmatic children chosen to undergo $\mathrm{pH}$ monitoring because of resistance to usual pulmonary therapies had abnormal studies [10]. Thus, $\mathrm{pH}$ monitoring teaches two major lessons: symptoms of GERD may be associated with physiologic esophageal acid exposure, and a significant percentage of patients with abnormal esophageal acid exposure have no or minimal clinical symptoms of reflux. The ongoing controversy is whether such asymptomatic patients can have extraesophageal symptoms that would respond to acid-reduction therapy. More likely, abnormal acid exposure defined by $\mathrm{pH}$ monitoring over a $24-\mathrm{h}$ period does not equate to GERD. There are also no data that $\mathrm{pH}$ studies allow the clinician to accurately predict the prognosis of GERD (natural history, risk of complications such as esophageal stricture or Barrett esophagus).

Intraluminal impedance is a new technology based on measuring changes in electrical conductivity between two rings connected to an alternating current (AC) generator [15]. Suspended in air the impedance catheter records high impedance values. The ions in a bolus of liquid (i.e. gastric contents) increase electrical conductivity and thereby decrease impedance. The potential advantage of intraluminal impedance measurements is the ability to detect nonacidic GER episodes. However, normal values in pediatric age groups have yet to be defined, analysis of tracings is time-consuming, and portable devices are unavailable for outpatient studies. Scintigraphy of a radiolabeled test meal also has the ability to detect acidic and non-acidic GER in the postprandial period as well as evaluate gastric emptying and occasionally detect aspiration of gastric contents during reflux. It too suffers from lack standardized techniques and absence of age-specific normative data.

The Society of Pediatric Radiology Position Statement in 1999 made two recommendations on the role of fluoroscopy in evaluating vomiting: (1) the primary role is to define whether there is a point of anatomic abnormality, but (2) the radiologist should also note whether there is GER, altered esophageal motility, or delayed gastric emptying. Clearly, fluoroscopy is superior to any other test in defining the anatomy of the upper GI tract. Indications for UGI series include bilious vomiting, forceful protracted vomiting, feeding difficulty/dysphagia, unexplained poor weight gain/weight loss, to assess the status of previous fundoplication, or simply to reassure the parent or physician of a child with chronic vomiting. The controversy lies in the role of fluoroscopy as a diagnostic test for GER. Unlike $\mathrm{pH}$ monitoring, there is no standard methodology. The volume of the barium meal varies, many times a nasogastric tube must be used in infants or toddlers who refuse to swallow barium, the duration for observation for spontaneous reflux varies between radiologists, and there is considerable variation between radiologists on the use of provocative maneuvers to elicit reflux. Certainly, extended observation or provocative maneuvers prolong fluoroscopy time. The majority of adult case series where $\mathrm{pH}$ monitoring is the standard for diagnosis report low sensitivity (16-50\%) and moderate specificity (77-94\%) of fluoroscopy for diagnosing reflux [16-19]. Provocative maneuvers, including abdominal compression, Valsalva maneuver, positional changes (upright, right lateral prone oblique, rolling side to side), leg lifting, coughing and water siphon test, improve sensitivity (44-92\%), but reduce specificity $(0-75 \%)$ [16-19].

Pediatric case series have reported that reflux is demonstrated at fluoroscopy in a large percentage of infants and children who are studied for any reason. The percentage of patients who have GER progressively diminishes with age (about $80 \%$ of infants less than 18 months decreasing to about $30 \%$ of adolescents between 12 and 18 years of age) [20]. GER is present in a number of children whose symptoms would not have suggested its presence. Finally, although many radiologists use grading systems to describe the height of reflux during fluoroscopy, there is no correlation between height of reflux and esophageal or extraesophageal symptoms of GERD, or prognosis or natural history of reflux [20]. Thus, the results of small case series evaluating fluoroscopy mimic the results found with $\mathrm{pH}$ monitoring. Although there is no doubt that fluoroscopy can demonstrate GER, this observation does not equate to GERD. Fluoroscopy time should not be prolonged to attempt to demonstrate GER during barium contrast radiography. There are no data to justify prolonging fluoroscopy time to perform provocative maneuvers to demonstrate reflux during barium contrast UGI series. It is important for radiologists to recognize how profoundly descriptive radiology reports impact on clinical management. Radiology reports should describe the presence or absence of reflux, but clearly state that demonstration of reflux during fluoroscopy does not equate to GERD.

\section{References}

1. Vandenplas Y, Sacre L (1987) Continuous 24 hour $\mathrm{pH}$ monitoring in 285 asymptomatic infants $(0-15$ months old). J Pediatr Gastroenterol Nutr 6:220-224 
2. Nelson SP, Chen EH, Syniar GM, et al (1997) Prevalence of symptoms of gastroesophageal reflux during infancy. A pediatric practice-based survey. Pediatric Practice Research Group. Arch Pediatr Adolesc Med 151:569-572

3. Rudolph CD, Mazur LJ, Liptak GS, et al (2001) Guidelines for evaluation and treatment of gastroesophageal reflux in infants and children: recommendations of the North American Society for Pediatric Gastroenterology and Nutrition. J Pediatr Gastroenterol Nutr 32:S1-S32

4. Mittal RK, Holloway RH, Penagini R, et al (1995) Transient lower esophageal sphincter relaxation. Gastroenterology 109:601-610

5. Nelson SP, Chen EH, Syniar GM, et al (2000) Prevalence of symptoms of gastroesophageal reflux during childhood: a pediatric practice-based survey. Pediatric Practice Research Group. Arch Pediatr Adolesc Med 154:150-154

6. Achem SR (1999) Endoscopy-negative gastroesophageal reflux disease. The hypersensitive esophagus. Gastroenterol Clin North Am 28:893-904

7. El-Serag HB, Gilger MA, Kuebler M, et al (2001) Extra-esophageal associations of gastroesophageal reflux disease in children without neurologic defects. Gastroenterology 121:1294-1299

8. Harding SM (2001) Gastroesophageal reflux, asthma, and mechanisms of interaction. Am J Med 111 [Suppl 8A]:8S-12S

9. Kahrilas PJ, Quigley EMM (1996) Clinical esophageal pH recording: a technical review for practice guideline development. Gastroenterology 110:1982-1996

10. Colletti RB, Christie DL, Orenstein SR (1995) Indications for pediatric esophageal $\mathrm{pH}$ monitoring. J Pediatr Gastroenterol Nutr 21:253-262

11. Hochman JA, Favaloro-Sabatier J (2005) Tolerance and reliability of wireless $\mathrm{pH}$ monitoring in children. J Pediatr Gastroenterol Nutr 41:411-415
12. Klauser AG, Schindlbeck NE, Muller-Lissner SA (1990) Symptoms of gastro-oesophageal reflux disease. Lancet 335:205-208

13. Gustafsson PM, Kjellman NI, Tibbling L (1992) A trial of ranitidine in asthmatic children and adolescents with and without pathological gastro-oesophageal reflux. Eur Respir J 5:201-206

14. Stordal K, Johannesdottir GB, Bentsen BS (2005) Acid suppression does not change respiratory symptoms in children with asthma and gastroesophageal reflux disease. Arch Dis Child 90:956-960

15. Rosen R, Nurko S (2004) The importance of multichannel intraluminal impedance in the evaluation of children with persistent respiratory symptoms. Am J Gastroenterol 99: $2452-2458$

16. Sellar RJ, DeCaestecker JS, Heading RC (1987) Barium radiology: a sensitive test for gastro-oesophageal reflux. Clin Radiol 38:303-307

17. Thompson JK, Koehler RE, Richter JE (1994) Detection of gastroesophageal reflux: value of barium studies compared with 24-hr pH monitoring. AJR 162:621-626

18. Johnston BT, Troshinsky MB, Castell JA, et al (1996) Comparison of barium radiology with esophageal $\mathrm{pH}$ monitoring in the diagnosis of gastroesophageal reflux disease. Am J Gastroenterol 91:1181-1185

19. Fransson SG, Sokjer H, Johansson KE, et al (1988) Radiologic diagnosis of gastro-oesophageal reflux by means of graded abdominal compression. Acta Radiol 29:45-48

20. Cleveland RH, Kushner DC, Schwartz AN (1983) Gastroesophageal reflux in children: results of a standardized fluoroscopic approach. AJR 141:53-56 